\title{
Post transplant Lymphoproliferative Syndromes
}

\author{
Jan Styczynski and Sebastian Giebel
}

\subsection{Definitions}

Post transplant lymphoproliferative disorders (PTLDs) constitute a heterogeneous group of lymphoproliferative diseases that occur in the setting of transplantation and result from the uncontrolled neoplastic proliferation of lymphoid or plasmacytic cells in the context of extrinsic immunosuppression after transplantation.

PTLD in HSCT setting are largely caused by latent Epstein-Barr virus (EBV, HHV-4), belonging to the herpesviruses family. It is one of the most common viruses in humans, with prevalence of $82-84 \%$ in overall population. EBV is associated with development of various diseases, which can be categorized as primary syndromes, EBVassociated tumors, and EBV-associated post transplant diseases: PTLD and other end-organ diseases (encephalitis/myelitis, pneumonitis, hepatitis, or hemophagocytic lymphohistiocytosis).

Comparably to other herpesviruses, there are two types of EBV infection: primary and recur-

J. Styczynski $(\triangle)$

Department of Pediatric Hematology and Oncology, Nicolaus Copernicus University Torun, Collegium Medicum, Bydgoszcz, Poland e-mail: jstyczynski@cm.umk.pl

S. Giebel

Department of Bone Marrow Transplantation and Onco-Hematology, Maria Sklodowska-Curie Memorial Cancer Center and Institute of Oncology, Gliwice, Poland rent. Primary EBV infection is diagnosed when $\mathrm{EBV}$ is detected (nucleic acid or serologically) in an EBV-naïve individual. Recurrent EBV-DNAemia (previously: latent infection) is diagnosed by detection of EBV-DNA in the blood, in a previously infected individual.

\subsection{Types of PTLD}

PTLD or end-organ EBV-associated post transplant disease can be diagnosed at the probable or proven level. Probable EBV disease is diagnosed in case of significant lymphadenopathy, hepatosplenomegaly, or other end-organ manifestations (without tissue biopsy, but in the absence of other documented cause) together with high EBVDNA-emia. Proven EBV disease (PTLD or other end-organ disease) is diagnosed in case of symptoms and/or signs from the affected organ together with the detection of EBV-encoded RNA by in situ hybridization (EBER-ISH) in a tissue specimen (immunohistochemistry for EBV proteins have good specificity but lower sensitivity; these proteins are variably expressed in PTLD biopsies). Histological WHO 2016 classification includes six types of morphological PTLD: plasmacytic hyperplasia, infectious mononucleosis-like, florid follicular hyperplasia, polymorphic, monomorphic (B-cell or T-/ NK-cell types), and classical Hodgkin lymphoma PTLD. 


\section{Atypical PTLD:}

- EBV-negative PTLD: A growing number of cases of EBV-negative PTLD have been reported, mainly in SOT recipients. These cases tend to present later ( $>5$ years after transplant) after transplant, and an increased risk is observed as long as 10 years after transplantation. These cases should be regarded as malignant lymphoma rather than as PTLD.

- T-lineage PTLD: T-PTLD is usually EBVnegative, and the relatively long latency between transplantation and T-PTLD onset may be explained by molecular events. The frequency of T-PTLDs ranges $4-15 \%$ of all PTLD cases. EBV is present in approximately one-third of T-PTLDs.

- Composite B-cell and T-cell lineage PTLD: Harboring both B- and T-cell clones either concurrently or successively in the same patient is extremely rare, and only a few cases have been reported in the literature, exclusively after SOT, with poor outcome.

\subsection{Pathogenesis}

The pathogenesis of PTLDs is a result of EBVinduced transformation of $\mathrm{B}$ cells in the setting of impaired anti-EBV cellular immunity due to iatrogenic IS and resulting in an outgrowth of EBVinfected B cells. GVHD prevention strategies that indiscriminately remove $\mathrm{T}$ cells from the graft increase the risk of PTLD.

Recurrent EBV infection preceding clinically overt PTLD is the consequence of viral latency, which is the stage in the viral life cycle in which no virions are produced, as opposed to the lytic stage. During viral latency of EBV, three associated patterns of viral protein expression, so-called latency programs, may be expressed. During infection of the B cell, these latency programs guide the $\mathrm{B}$ cell through the germinal center reaction pushing it toward the resting memory cell stage. Different latency proteins are implicated in EBV-driven lymphomagenesis demonstrated by the expression of a particular latency program in different lymphoma subtypes.

$B$ cells in PTLD express a number of latency proteins which are highly immunogenic and are vigorously targeted by $\mathrm{T}$ lymphocytes in immu- nocompetent hosts. This viral gene program in EBV-PTLD is called type III of latency and is different and less immunogenic than in other EBVrelated diseases with type I or II of latency.

EBV plays also an important role in pathogenesis and epidemiology of acute and chronic GVHD. As B cells also play a role in the pathophysiology of chronic GVHD, and B cells are stimulated into activity by EBV infection, and B-cell recovery occurs usually after day +100 , the impact of EBV-infected B cells is stronger for development of chronic, and to less extent, acute GVHD.

\subsection{Clinical Manifestations}

Lymphadenopathy and fever are the most common symptoms of EBV-PTLD. Rare EBVassociated PTLD manifestations, also referred as EBV end-organ disease, include encephalitis/ myelitis, pneumonitis, hepatitis, and hemophagocytic lymphohistiocytosis.

\begin{tabular}{|c|c|}
\hline Time to PTLD & $\begin{array}{l}\text { Median time of PTLD } \\
\text { development: } 2-4 \text { months } \\
6 \% \text { PTLD cases are diagnosed } \\
\text { within first month } \\
90 \% \text { diagnosed within first } 6 \\
\text { months after HSCT } \\
\text { Rarely: }>5 \text { year post-HSCT (more } \\
\text { likely representing lymphoma) }\end{array}$ \\
\hline $\begin{array}{l}\text { Incidence of } \\
\text { EBV-DNA-emia }\end{array}$ & $\begin{array}{l}\text { Median: } 29.4 \% \text { (range: } 0.1-63 \% \text { ) } \\
\text { Highest in: MUD/MMUD-HSCT, } \\
\text { haplo-HSCT without PTCy } \\
\text { Lowest in: haplo-HSCT with PTCy } \\
\text { (post-HSCT Cy) }\end{array}$ \\
\hline $\begin{array}{l}\text { Incidence of } \\
\text { PTLD }\end{array}$ & $\begin{array}{l}\text { All allo-HSCT: } 3.22 \% \\
\text { MFD-HSCT: } 1.16 \% \\
\text { MMFD-HSCT: } 2.86 \% \\
\text { MUD-HSCT: } 3.97 \% \\
\text { MMUD-HSCT: } 11.24 \% \\
\text { CBT: } 4.06 \% \\
\text { Auto-HSCT without TCD: } \\
\text { casuistic }\end{array}$ \\
\hline Target organs & $\begin{array}{l}\text { Frequently: lymph nodes } \\
\text { Rarely: CNS, GI tract, lungs, liver }\end{array}$ \\
\hline
\end{tabular}

${ }^{a}$ Level of donor match determined locally as $8 / 8$ or $10 / 10$

\subsection{Diagnosis}

The diagnosis of EBV-PTLD must be based on symptoms and/or signs consistent with PTLD 
together with detection of EBV by an appropriate method applied to a specimen from the involved tissue. Definitive diagnosis of EBVPTLD requires noninvasive and invasive techniques (biopsy and histological examination).

\section{Noninvasive diagnostic methods \\ Quantitative determination of EBV-DNA-emia ${ }^{\mathrm{a}}$ \\ Imaging: CT or PET-CT ${ }^{\text {b }}$ (for avid structures, localized in the lymph nodes, spleen, liver, GI tract, skin, lungs, bone, BM) or MRI (in CNS disease and non-avid histologies) \\ Invasive diagnostic methods \\ Biopsy of the lymph node and/or other suspected sites Endoscopy: when GI symptoms \\ Histological examination \\ (a) Detection of viral antigens or in situ hybridization for EBER (EBV-encoded RNA) transcripts \\ (b) Immunohistochemistry \\ (c) Flow cytometry for B cell, T cell, and plasma cell lineage-specific antigens}

${ }^{a}$ Monitoring EBV-DNA-emia: Serial quantitative measurement of EBV viral load post transplant is currently the method of choice for early detection and monitoring progression and response to treatment of EBVPTLD. Although the value of the viral load in PTLD risk assessment is uncertain, it is recommended to begin the screening in patients with risk factors after hematological recovery and no later than 4 weeks after the day of HSCT. In EBV-DNA-negative patients, frequency of screening should be once a week, while in patients with rising EBV-DNA-emia, more frequent sampling might be considered, as the calculated doubling time for EBV might be as short as $56 \mathrm{~h}$. The screening should be continued at least 4 months in high-risk patients. Longer monitoring is recommended in patients considered to have poor T-cell reconstitution, with severe GVHD, after haplo-HSCT, with the use of TCD, after conditioning with ATG/alemtuzumab, or in those having experienced an early EBV reactivation

bPET imaging: By definition, PTLD is a neoplastic lymphoproliferation. Malignant lymphomas have the ability to metabolize $18 \mathrm{~F}$-fluorodeoxyglucose (FDG), which is used by PET imaging. In most cases, PTLD has FDG-avid histology; thus FDG-PET is an important diagnostic tool for this disease

\subsection{Risk Factors}

Risk of development of PTLD is essentially proportional to the degree of T-cell depletion/impairment, and this should be regarded as the principal risk factor. Thus, the type of donor and type of conditioning have secondary value as risk factors. Since in HSCT setting PTLD usually origi- nates from donor, the risk of PTLD is obviously higher when the donor is seropositive. Risk factors for PTLD in match family donor (MFD) transplants include TCD ex vivo or in vivo, EBV serology mismatch between donor and recipient, and splenectomy. ECIL-6 classified HSCT patients into three groups of the risk for EBVPTLD: low, standard, and high risk.

\begin{tabular}{l|l} 
Risk group & Patients \\
High & $\begin{array}{l}\text { MUD/MMUD } \\
\text { Alternative donors including CBT } \\
\text { MFD-HSCT with at least one risk factor }\end{array}$ \\
Standard & $\begin{array}{l}\text { MFD-HSCT without risk factors } \\
\text { Haplo-PTCy-HSCT }\end{array}$ \\
\hline Low & Auto-HSCT
\end{tabular}

\subsection{Grading}

No grading system currently exists for PTLD. It seems that apart from the findings from biopsy material with a histological examination; the diagnostic criteria of tissue involvement in PTLD should be consistent with those for lymphoma (the Ann Arbor and the Lugano classifications). Nowadays, the use of FDG-PET-CT has emerged as an important imaging tool for PTLD diagnosis and staging.

Possible staging of PTLD:

- Clinical end-organ staging: nodal vs. extranodal disease

- Clinical severity staging: limited (unifocal) vs. advanced (multifocal) disease

- ECIL-6 staging (based on the Lugano lymphoma classification by PET-CT imaging): limited (stages I-II), advanced forms (stages III-IV)

\subsection{Treatment}

\subsubsection{Prevention: Donor and Recipient Issues}

As EBV might be transmitted with the graft, selection of EBV-seronegative donor might be beneficial for EBV-seronegative recipient, if possible. For EBV-seropositive patients, selection of 
an EBV-seropositive donor might be justified, as transmission of EBV-specific CTLs outweighs the risk of transmission of EBV-positive B cells from the donor.

\subsubsection{Treatment Strategies}

PTLD has to be regarded as disseminated disease at diagnosis. This is because of the involvement of lymphoid tissue, which is localized throughout the whole body. Therapeutic approaches applied in the prevention and treatment of EBV-PTLD include administration of RTX, reduction of immunosuppression (RIS), use of EBV-CTL, DLI, and chemotherapy, while other methods have only historical value. No antiviral drug is currently effective against EBV. There are three major approaches to EBV infection after HSCT: prophylaxis, preemptive therapy (also known as preemptive prophylaxis), and treatment of established EBV-PTLD.

\subsubsection{Prophylaxis}

Prophylaxis of EBV disease is defined as drug or cellular therapy given to an asymptomatic EBVseropositive patient to prevent EBV-DNA-emia. This strategy is administered rarely, with the use of rituximab (RTX) or EBV-CTL.

The rationale for prophylactic use of RTX before or early after allo-HSCT is B-cell depletion. Prophylactic use of post transplant RTX reduced the risk of EBV-DNA-emia, with no impact on PTLD incidence, TRM, or OS in comparison to preemptive therapy.

The prophylactic use of EBV-CTLs resulted in excellent efficacy in patients at a high-risk group for EBV-PTLD. The obstacle for the use of this approach is limited availability of CTLs for most transplant centers.

Low risk of EBV-DNA-emia and EBV-PTLD was observed after the use of PT-CY and SIR for GVHD prophylaxis.

\subsubsection{Preemptive Therapy}

Drugs or cellular therapy is given to a patient with EBV-DNA-emia in order to prevent EBV disease.
Monitoring for EBV-DNA-emia is essential in all patients with risk factors for EBVPTLD. Significant EBV-DNA-emia without clinical symptoms of disease in high-risk patients for EBV-PTLD is usually an indication for preemptive therapy.

The goal is to obtain a negative EBV PCR or EBV-DNA-emia below the initial threshold without relapse.

Usually, EBV-DNA-emia occurs prior to the onset of clinical symptoms. There is a correlation between rising or high EBV-DNA-emia in PB and the development of EBV-PTLD, but this is not the rule.

Currently available data does not allow for the determination of an unambiguous EBV-DNA threshold value for the diagnosis of EBV-PTLD or other end-organ EBV disease in HSCT patients.

Apart from EBV-DNA value, also the kinetics of a rising EBV-DNA-emia, together with an assessment of an individual patient's immune function, are very important when appraising the need for preemptive therapy. Local experience based on correlation of clinical and laboratory data might be a rationale for center-specific cutoff value.

The primary method for preemptive therapy includes RTX, once weekly until EBV-DNA-emia negativity. Usually, 1-2 doses of RTX are sufficient. RTX should be combined with reduction of the IS (RIS), if possible. A contraindication for RIS is severe, uncontrolled acute, or chronic GVHD. This approach might have additional benefit of RTX administration, as RTX possibly reduces the risk of acute/chronic GVHD. Donor or third-party EBV-CTL is another option, although it is not widely available.

\subsubsection{Treatment of Established EBV-PTLD}

Treatment of established EBV-PTLD means therapeutic interventions for patients with probable or proven EBV disease. Due to the consequential risk of a rapidly growing high-grade lymphoid tumor, together with the potential for EBV to cause rapid MOF, therapy should be implemented as soon as possible. 
For first-line therapy, three options are recommended: (a) RTX, $375 \mathrm{mg} / \mathrm{m}^{2}$, once weekly; (b) RIS, if possible, usually together with administration of RTX; (c) adoptive immunotherapy with cellular therapy with in vitro generated donor or third-party EBV-CTL, if available.

For the second-line therapy, in case of RTX failure: (a) cellular therapy (non-specific DLI or specific EBV-CTLs, if available); (b) chemotherapy \pm RTX. Unselected DLI from EBVpositive donor is used in order to restore broad T-cell reactivity, including EBV-specific responses.

Not recommended: IVIg, interferon, and antiviral agents should not be used for therapy of PTLD.

\subsubsection{Results of Anti-EBV-PTLD Therapy}

\begin{tabular}{|l|l|l|}
$\begin{array}{l}\text { Treatment } \\
\text { strategy }\end{array}$ & $\begin{array}{l}\text { Preemptive } \\
\text { therapy }(\%)\end{array}$ & $\begin{array}{l}\text { Therapy of } \\
\text { PTLD (\%) }\end{array}$ \\
\hline $\begin{array}{l}\text { RTX } \\
\text { RTX + RIS }\end{array}$ & 90 & 65 \\
\hline EBV-CTL & $94-100$ & 78 \\
\hline RIS & 68 & $71-75$ \\
\hline DLI & & 61 \\
\hline Chemotherapy & & $58^{\mathrm{a}}$ \\
\hline $\begin{array}{l}\text { Antivirals } \\
\text { (cidofovir) }\end{array}$ & & $26^{\mathrm{a}}$ \\
\hline
\end{tabular}

$R T X$ rituximab, RIS reduction of immunosuppression ${ }^{a}$ With other therapies

\subsubsection{Treatment in CNS Disease}

CNS localization of PTLD is a special form of the disease, due to the risk of neurological consequences even in case of successful eradication of EBV from CNS. No standard therapy has been accepted up to date.

Possible therapeutic options include (a) RTX, either systemic or intrathecal; in the latter case, dose of RTX was 10-30 mg in 3-10 mL saline administered weekly; (b) T-cell therapy with EBV-CTLs; (c) radiotherapy; (d) chemotherapy \pm RTX according to primary CNS lymphoma protocols based on high dose of MTX \pm Ara-C.

\subsubsection{Criteria of Response to Therapy in EBV-PTLD}

The treatment goal in EBV-PTLD setting is the resolution of all signs and symptoms of PTLD together with negative EBV-DNA-emia.

The response to RTX therapy can be identified by a decrease in EBV-DNA-emia of at least $1 \mathrm{log}$ of magnitude in the first week of treatment.

Positive prognostic factors for outcome to RTX therapy include age below 30 years, underlying non-malignant disease, no acute GVHD $\geq$ II, RIS at time of PTLD diagnosis, and decrease of viral load after 1 or 2 weeks of therapy.

Complete remission of PTLD can be defined as resolution of all symptoms of PTLD, including clearance of EBV-DNA-emia. Partial response of PTLD can be stated with the decrease of at least $50 \%$ of initial changes, including decrease of EBV-DNA-emia.

The response to therapy can be confirmed by achievement of a PET-negative complete remission for avid lymphomas and CT/MRI for nonavid histologies or CNS localization.

\section{Key Points}

- Definition: PTLD results from an uncontrolled neoplastic proliferation of lymphoid or plasmacytic cells in the context of extrinsic IS after HSCT transplantation. PTLDs in HSCT setting are largely caused by latent EBV. Risk factors for EBV-PTLD are proportional to the degree of T-cell impairment.

- Diagnosis: Should be based on invasive techniques including biopsy of the lymph node and/or other sites suspected for EBV disease. Noninvasive diagnostic methods have accessory value and include the quantitative determination of EBV-DNA-emia in blood, plasma or serum, and PET-CT/CT/MRI.

- Management strategies: Prophylaxis, preemptive treatment, and therapy of established EBV-PTLD. Therapeutic 
approaches include administration of rituximab, RIS, use of EBV-CTL, or DLI and chemotherapy, while other methods have only a historical value.

- EBV-DNA-emia threshold value: No value determines diagnosis of EBVPTLD or other end-organ EBV disease in HSCT patients. In order to initiate preemptive therapy, transplant centers should use own threshold values of EBV-DNA-emia.

- Outcome: Administration of rituximab results in a positive outcome for over $90 \%$ of patients treated preemptively and over $65 \%$ when it is used as targeted therapy for EBV-PTLD. RIS when applied in combination with rituximab: over $80 \%$. The use of EBV-CTLs: $>90 \%$ of patients treated preemptively and approximately $75 \%$ in therapy of EBV-PTLD.

\section{Recommended References}

Bollard CM, Heslop HE. T cells for viral infections after allogeneic hematopoietic stem cell transplant. Blood. 2016;127:3331-40.

Czyzewski K, Styczynski J, Krenska A, et al. Intrathecal therapy with rituximab in central nervous system involvement of post-transplant lymphoproliferative disorder. Leuk Lymphoma. 2013;54:503-6.
Dharnidharka VR, Webster AC, Martinez OM, et al. Posttransplant lymphoproliferative disorders. Nat Rev Dis Primers. 2016;2:15088.

Dierickx D, Habermann TM. Post-transplantation lymphoproliferative disorders in adults. $\mathrm{N}$ Engl $\mathrm{J}$ Med. 2018;378:549-62.

Dierickx D, Tousseyn T, Requile A, et al. The accuracy of positron emission tomography in the detection of posttransplant lymphoproliferative disorder. Haematologica. 2013;98:771-5.

Styczynski J. Managing post-transplant lymphoproliferative disorder. Expert Opin Orphan Drugs. 2017;5:19-35.

Styczynski J, Gil L, Tridello G, et al. Response to rituximab-based therapy and risk factor analysis in EBV-related lymphoproliferative disorders after hematopoietic stem cell transplantation in children and adults: a study from the Infectious Diseases Working Party of the European Group for Blood and Marrow Transplantation. Clin Infect Dis. 2013;57: 794-802.

Styczynski J, Tridello G, Gil L, et al. Impact of donor Epstein-Barr virus serostatus on the incidence of graft-versus-host disease in patients with acute leukemia after hematopoietic stem-cell transplantation: A study from the Acute Leukemia and Infectious Diseases Working Parties of the European Society for Blood and Marrow Transplantation. J Clin Oncol. 2016a;34:2212-20.

Styczynski J, van der Velden W, Fox CP, et al. Management of Epstein-Barr Virus infections and post-transplant lymphoproliferative disorders in patients after allogeneic hematopoietic stem cell transplantation: Sixth European Conference on Infections in Leukemia (ECIL-6) guidelines. Haematologica. 2016b;101:803-11.

Uhlin M, Wikell H, Sundin M, et al. Risk factors for Epstein-Barr virus-related post-transplant lymphoproliferative disease after allogeneic hematopoietic stem cell transplantation. Haematologica. 2014;99:346-52.

Open Access This chapter is licensed under the terms of the Creative Commons Attribution 4.0 International License (http://creativecommons.org/licenses/by/4.0/), which permits use, sharing, adaptation, distribution and reproduction in any medium or format, as long as you give appropriate credit to the original author(s) and the source, provide a link to the Creative Commons license and indicate if changes were made.

The images or other third party material in this chapter are included in the chapter's Creative Commons license, unless indicated otherwise in a credit line to the material. If material is not included in the chapter's Creative Commons license and your intended use is not permitted by statutory regulation or exceeds the permitted use, you will need to obtain permission directly from the copyright holder. 